\title{
Zonal failure structure near the deep openings
}

\author{
VV Makarov Far Eastern Federal University, Russia
}

LS Ksendzenko Far Eastern Federal University, Russia

NA Opanasiuk Far Eastern Federal University, Russia

AM Golosov Far Eastern Federal University, Russia

\begin{abstract}
Rock mass failure at high depth near deep openings often has a zonal character (Makarov et al. 2016). The mechanism of this phenomenon consists in the periodical character realisation of stresses in surrounding rock mass and development of tensile macrocracks at the places (zones) of maximum tangential stresses (Guzev \& Makarov 2007). A mathematical model of the highly stressed rock massifs is developed on the base of the defective media mechanics and non-equilibrium thermodynamics principals (Makarov et al. 2013). To determine the mathematical model parameters of the rock mass zone destruction in the great depths conditions, we proposed a method founded on the experiments with the rock samples. Relationships between the width of cracking zones and rock massif strength property have been obtained.
\end{abstract}

Keywords: rock massif, opening, zonal failure, mathematical model

\section{Introduction}

Failure conditions can take place in the boundary parts of openings at high depths of excavation and wall drilling. In some cases, the failure has a zonal character (Figure 1), where tensile macrocrack zones alternate with relatively monolithic rock mass (Adams \& Jager 1980; Shemjakin et al. 1986; Qian et al. 2009; Lisjak et al. 2012; Cumming-Potvin et al. 2016). Many attempts have been made to describe the zonal character of rock mass failure near openings based on classical mechanics (Metlov et al. 2002; Odintsev 1996; Reva \& Tropp 1995; Shemjakin et al. 1987). But no one has been able to explain all the properties of zonal failure structures without the introduction of new assumptions in every new case.

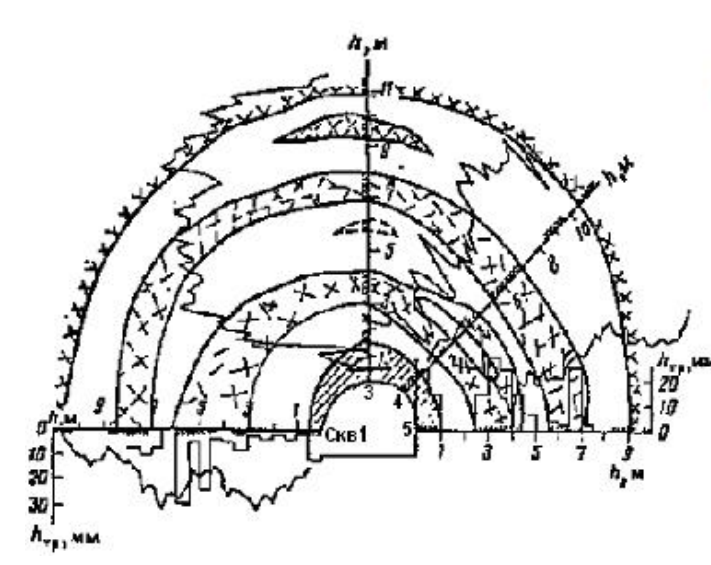

(a)

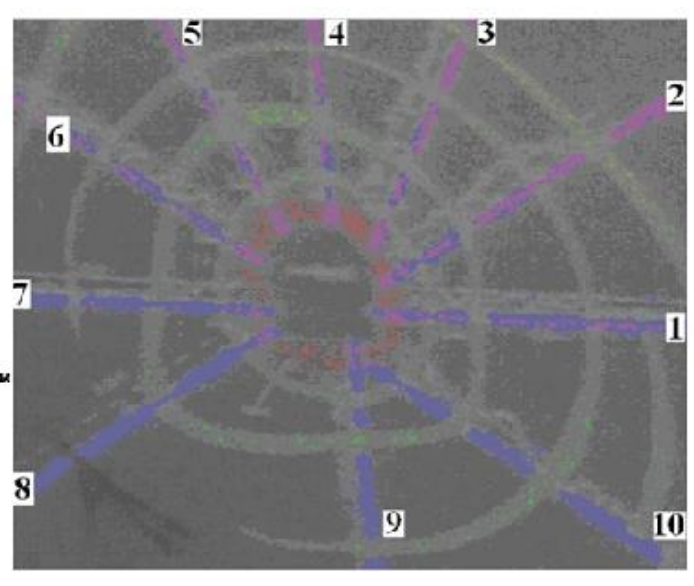

(b)

Figure 1 (a) The nature of the zonal failure rock massif around a single opening, according to Shemjakin et al. (1986); (b) zonal failure rock massif around a single opening according to Qian et al. (2009) 
Some attempts to describe zonal failure near openings have been done with the finite element method/discrete element method (FEM/DEM) (Lisjak et al. 2012) where the block caving method of excavation has been applied. But FEM/DEM is founded on the classical theory of the continuous media where the compatibility conditions have been met and cannot be applied to the highly stressed rock mass with the zone failure description purposes (Reva \& Tropp 1995; Mirenkov 2014).

Another approach is taken place with the problem of physical modelling on the centrifuges (Cumming-Potvin et al. 2016). The authors ignore the principals of the highly stressed media modelling, which have been achieved after the same type of research in VNIMI (the State Geomechanical Institute, Leningrad, Russia) (Glushihin et al. 1991), and have interpreted the failure of the continuous media in the one radius distance around the opening as the 'zones character' which does not satisfy the experiment's results in situ.

Recently, a new gauge theory has been applied to solids to describe the whirl fields of plasticity in high energy conditions (Kadich \& Edelen 1983; Panin 1990; Panin et al. 1990). The main principle of gauge theory is incompatibility of the deformation in solid media. But it does not apply to the zonal failure phenomenon of rock masses near openings. In this paper we demonstrate an example of a description of the phenomenon by employing the gauge mathematical model.

The description can be done from the position of consideration of the rock mass hierarchical block systems (Xu 2009). A rock sample in this conception is shown as the first level of a hierarchical system and the rock mass on the opening scale corresponds with the second one.

\section{$2 \quad$ Mathematical model and task solution}

Rock at a high depth is modelled by a faulted structure which is far from the state of thermodynamical equilibrium; it is in constant all-around compression. The problem is formulated as a two-dimensional circular opening in an infinite medium with compressive gravitational stress applied at an infinite boundary. (Appendix A). Internal body forces are not taken into account.

At the opening boundary the rock mass undergoes considerable destruction; therefore the damage parameter $R$ should not equal zero. Assuming that all zones of rock destruction are equivalent and are of the same origin, we introduce the extremum of the function condition in the boundary $R(r)$ and the following zones of destruction. Therefore boundary conditions for the function $R(r)$ are:

$$
\left.R^{\prime}(r)\right|_{r=r_{0}}=0,\left.R^{\prime}(r)\right|_{r=r^{*}}=0
$$

where:

$$
r^{*}=\text { parameter, determined experimentally. }
$$

The equation for the first invariant of stresses $\sigma=\sigma_{z z}+\sigma_{r r}+\sigma_{\varphi \varphi}$ is:

$$
\Delta \sigma=\frac{E}{2(1-v)} R, \sigma \rightarrow 2(1+v) \sigma_{\infty}, r \rightarrow \infty
$$

where:

$$
\begin{aligned}
& R=\text { the determined function. } \\
& E \quad=\text { the modulus of elasticity. } \\
& v \quad=\text { the Poisson ratio. }
\end{aligned}
$$


Solving Equation (2) gives expressions for the stress components:

$$
\begin{gathered}
\sigma_{r r}=\sigma_{\infty}\left(1-\frac{r_{0}^{2}}{r^{2}}\right)-\frac{E}{2\left(1-v^{2}\right) \gamma^{3 / 2}} \cdot \frac{1}{r}\left[a J_{1}(\sqrt{\gamma} r)+b N_{1}(\sqrt{\gamma} r)+c K_{1}(\sqrt{\gamma} r)\right] ; \\
\sigma_{\varphi \varphi}=\sigma_{\infty}\left(1+\frac{r_{0}^{2}}{r^{2}}\right)-\frac{E}{2\left(1-v^{2}\right) \gamma}\left[a J_{0}(\sqrt{\gamma} \cdot r)+b N_{0}(\sqrt{\gamma} \cdot r)-c K_{0}(\sqrt{\gamma} \cdot r)\right]+ \\
+\frac{E}{2\left(1-v^{2}\right) \gamma^{3 / 2}} \cdot \frac{1}{r}\left[a J_{1}(\sqrt{\gamma} \cdot r)+b N_{1}(\sqrt{\gamma} \cdot r)+c K_{1}(\sqrt{\gamma} \cdot r)\right],
\end{gathered}
$$

where:

$=$ the distance from the centre of the opening to a selected point in the rock mass.

\section{Stress calculation and criteria of failure for non-classical boundary conditions and task solution}

Stresses surrounding the opening are of oscillating character (Figure 2).

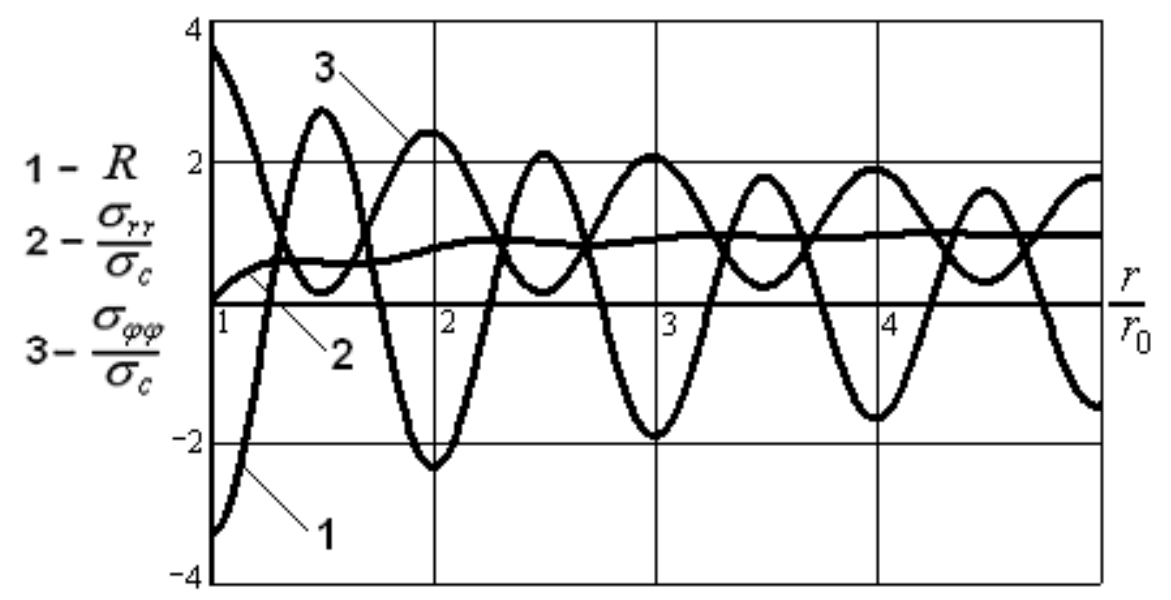

Figure 2 Oscillating character of the stresses and $R$ - functions in the rock mass surrounding the underground opening

The zones of failure appear in the areas where the conditions of crack propagation under compression are met:

$$
K_{I}=(\pi \cdot l)^{1 / 2} \cdot\left(\gamma_{1} \cdot \sigma_{1}^{0}-\gamma_{3} \cdot \sigma_{3}^{0}\right) \geq K_{I c}
$$

where:

$l=$ the half-length of fracture faults of the rock mass and is assumed to be equal to the minimum half-length of a tensile macrocrack which is unstable in stress conditions ( $\mathrm{m})$.

$\sigma_{1}^{0}, \sigma_{3}^{0}=$ the maximum and minimum of major stresses, respectively ( $\mathrm{MPa}$ ).

$\gamma_{1}, \gamma_{3}=$ empirical factors.

$K_{I} \quad=$ the stress intensity factor $\left(\mathrm{MPa} * \mathrm{~m}^{1 / 2}\right)$.

$K_{I c} \quad=$ the fracture toughness of rock material $\left(\mathrm{MPa} * \mathrm{~m}^{1 / 2}\right)$. 
As a criterion function the following dependence is taken:

$$
K(r)=K_{I} / K_{I c} .
$$

At $K_{I} / K_{I c}<1$ there is no failure around the opening; at $K_{I} / K_{I c} \geq 1$ the fracture starts to appear. The criterion function, as well as the stress functions and $R$, has an oscillating character (Figure 3).

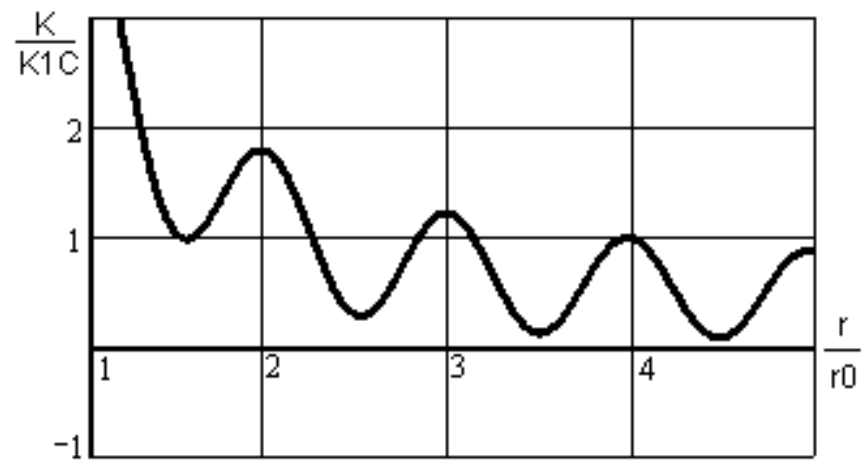

Figure 3 Character of criterion function

\section{$4 \quad$ Parameters of the model}

The research on rock mass zonal failure was carried out for an unlined opening. In order to run the calculations, algorithms and programs were developed that included formulas for calculating the damage function $R(r)$, stresses, and criterion function $K_{r}(r)$. On the basis of the developed programs the experiment was carried out with three types of parameters being analysed.

The first type included parameters of model $\gamma, \mathrm{c}$, which are determined from the experiment taking into account the all-around compression at a high depth. The second type includes parameters that characterise the mechanical properties of rock mass: $E$, the modulus of elasticity (MPa); $v$, the Poisson ratio; $\sigma_{c}$, the uniaxial compression strength (MPa); and the value of gravitational stresses in the rock mass, $\sigma_{\infty}(\mathrm{MPa})$. The third type includes parameters in Equation (4) which characterise the cracking structure of the rock mass: the half-length of rock fracture faults $l$ and the fracture toughness of rock material $K_{I c}$, and the coefficients $\gamma_{1}, \gamma_{3}$ (below the dependence $\gamma_{3} / \gamma_{1}$ is used).

Parameter $\gamma$ can be determined using the procedure of statistical analysis of the natural research results of the zonal failure process in deposits in Russia (the Far Eastern part, Siberia, Donbass) and China. A linear character of dependence between the relative distance from the opening contour to the middle point of the first failure zone and uniaxial strength of the rock was determined:

$$
r^{*} / r_{0}=0.0083 \sigma_{c}+0.748
$$

where:

$r^{*}=$ the distance from the opening contour to the middle point of the first failure zone which has been found from experimental data $(m)$.

The relation between parameters $\gamma$ and $r^{*} / r_{0}$ is linear as can be seen from Table 1. 
Table 1 Meanings of the parameter $\gamma$ of the model

\begin{tabular}{lllll}
\hline $\begin{array}{l}\text { Middle part of the l-st zone, } \\
\mathbf{r}^{*}=\mathbf{- r} / \mathbf{r} \mathbf{0}\end{array}$ & $\mathbf{r} \mathbf{0}=\mathbf{1 . 7 5}$ & $\mathbf{r} \mathbf{0}=\mathbf{2 . 0}$ & $\mathbf{r} \mathbf{0}=\mathbf{2 . 5}$ & $\mathbf{r} \mathbf{0}=\mathbf{3 . 0}$ \\
\hline 0.7 & 26.486 & 20.279 & 12.978 & 9.012 \\
\hline 0.8 & 20.313 & 15.552 & 9.953 & 6.912 \\
\hline 0.9 & 16.080 & 12.311 & 7.879 & 5.471 \\
\hline 1.0 & 13.050 & 9.991 & 6.394 & 4.440 \\
\hline
\end{tabular}

According to recent research the rock mass can be shown as a hierarchical block medium (Guzev \& Makarov 2007; Guzev \& Paroshin 2000; Guzev et al. 2005; Kadich \& Edelen 1983; Makarov 2004, Makarov et al. 2007). When the physical character of failure on the neighbouring hierarchical levels is the same, the macrodefect size of the lower level can be determined as a mesodefect of the corresponding higher level (Guzev \& Makarov 2007; Guzev \& Paroshin 2000). So this low is reflected in the conservation shear-tensile character of the rock failure on the neighbouring levels of samples and mass in conditions of high stress (Makarov et al. 2007).

The algorithm for the determination of the mathematical model's parameters consists of the next steps:

After the rock sample strength reaches the limit of strength $\sigma_{C}$, the limit of residual strength $\sigma_{C}^{\text {res }}$, Young's modulus $E$, and Poisson's ratio $v$ are determined. Then by using Equation (6) the emplacement of the first failure zone's middle point $r^{*} / r_{0}$ can be found. Then after the substitution of these data in Table 1, the first correction parameter of the mathematical model $\gamma$ can be determined.

The maximum diameter $\mathbf{r}$ of the rock sample minerals $d_{\max }$ and maximum mesocrack disclosure $h_{*} \approx d_{\max }$ are determined and after that the minimum half-length of the tensile mesocrack

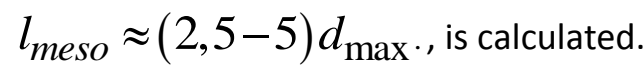

The critical half-length of the tensile macrocrack in the sample is determined by the formula $l_{*}=\frac{h_{*} \cdot E}{4\left(1-v^{2}\right) \gamma_{1} \cdot \sigma_{c}}$, and the stress intensity factor of the rock mass is calculated by the formula $K_{I}^{\text {mass }}=\gamma_{1} \cdot \sigma_{c}^{r e c} \cdot \sqrt{\pi l_{\text {meso }}^{\text {mass }}}$.

Then, the stress intensity factors of both the rock sample and the rock mass are written in the equation of equality and the parameter of the model ' $C$ ' is determined as a result of the calculation. For this purpose, the equation of equality is applied:

$$
K_{I}^{\text {mass }}=\gamma_{1} \cdot \sigma_{c}^{r e c} \cdot \sqrt{\pi l_{\text {meso }}^{\text {mass }}}=K_{1}=(\pi l)^{1 / 2}\left(\gamma_{1} \sigma_{\varphi \varphi}-\gamma_{3} \sigma_{r r}\right)
$$

where:

$\mathrm{C}=$ parameter used in the right-hand part of Equation (7) (see Appendix).

Programs for the determination of the last destruction zone (for lined and unlined openings: B1 and B2 respectively) and programs for calculation of the radial length of fracture zones (for lined and unlined openings: $\mathrm{Cl}$ and $\mathrm{C} 2$ respectively) were developed. Program charts with a brief description were also developed. The patterns of the changes in the zonal structure of rock mass failure depending on various factors were obtained. 
The main parameters of the zone structure were identified: the number of zones of failure, the location of the furthest fracture zone from the opening boundary (the last zone of failure); the creation of relative critical stresses of failure zones; and the value of the radial length of failure zones.

\section{$5 \quad$ Results}

As a result of the modelling experiment on the basis of the adopted mathematical model we determined that the parameters of the zonal structure depend slightly on the values of the elastic modulus of rocks $E$ and the Poisson ratio $v$. This conclusion corresponds to the data obtained during laboratory studies (when the elasticity varies 10 times, the critical stresses of zone creation change by $2-5 \%$ on average). The research on fracture zones was carried out for rather solid rock $\left(\sigma_{c}=150 \mathrm{MPa}\right)$ and for weak rock ( $\sigma_{c}=15 \mathrm{MPa}$ ). In order to run the calculations, algorithms and programs were developed that included formulas for calculating the expressions of defectiveness $R(r)$, stress, and the criterion function $K(r)$.

The results of the solid rock case study are demonstrated by the application of the developed method to the problem of zonal failure in the Nikolaevskij ore mine (Dalnegorsk, Russia). The forecasted depth of development of the cracking zone is shown in Table 2.

Table 2 Forecasted depth of development of zonal failure in the Nikolaievskij ore mine

\begin{tabular}{|c|c|c|c|c|}
\hline Number of failure zones & $\mathbf{I}$ & II & III & IV \\
\hline $\begin{array}{l}\text { Relative critical stress of zone } \\
\text { formation }\end{array}$ & 1.3 & 2.3 & 2.9 & 3.3 \\
\hline Depth of zone appearance $(\mathrm{m})$ & 520 & 920 & 1,160 & 1,320 \\
\hline $\begin{array}{l}\text { Relative critical stress of zone } \\
\text { formation }\end{array}$ & 1.3 & 2.3 & 2.9 & 3.3 \\
\hline Depth of zone appearance $(\mathrm{m})$ & 520 & 920 & 1,160 & 1,320 \\
\hline
\end{tabular}

The amplitude parameter ' $C$ ' is dependent on the modulus of deformation and the Poisson ratio of the rock mass (Figure 4).
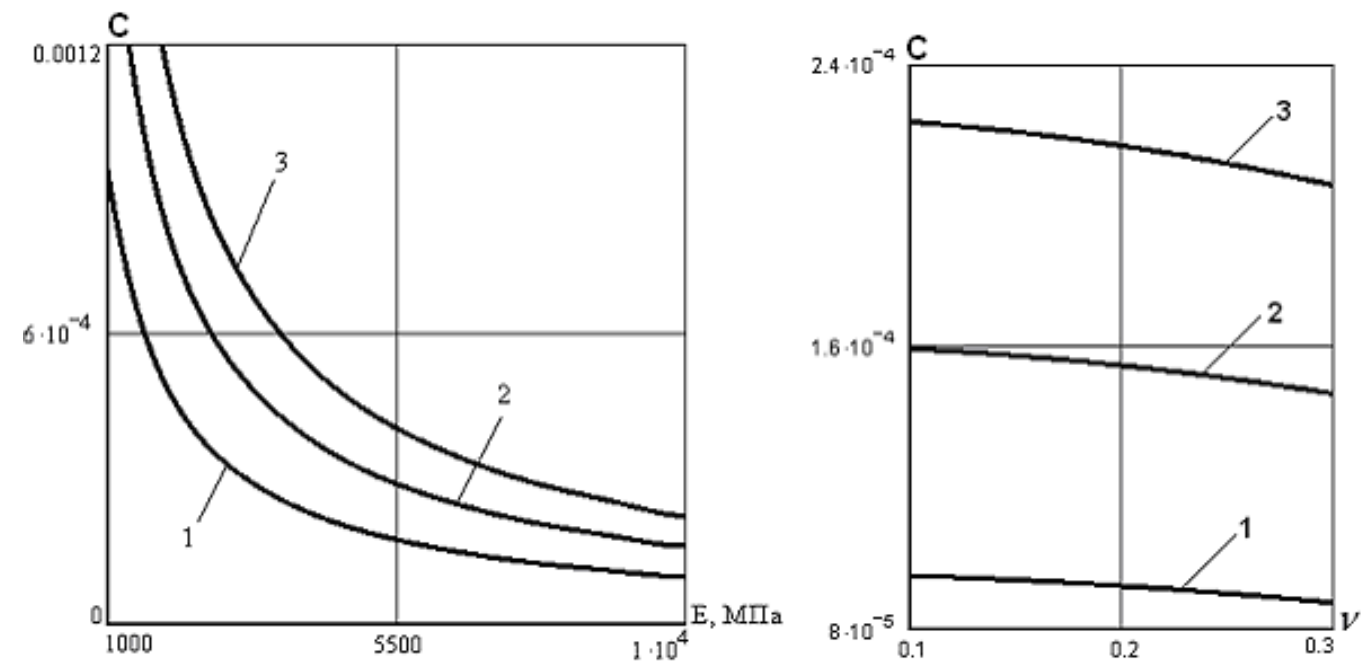

Figure 4 The relationships of the model amplitude parameter with the deformation modulus $E$ and Poisson's ratio $v$ under conditions of different values of rock mass failure strength: $1-K_{I C}=1.5 \mathrm{MPa} \cdot \mathrm{m}^{1 / 2}, 2-K_{I C}=2.0 \mathrm{MPa} \cdot \mathrm{m}^{1 / 2}, 3-K_{I C}=2.5 \mathrm{MPa} \cdot \mathrm{m}^{1 / 2}$ 
The precision of the correlation between theory and experiment has been estimated by comparing the results of in situ measurement of the radial displacements near the openings at high depth (Nikolaevskij ore mine) with the model calculation results (Figure 5). It was determined that the difference between the forecasted and measured data was no more than $50 \%$ in the four radius field around the opening.

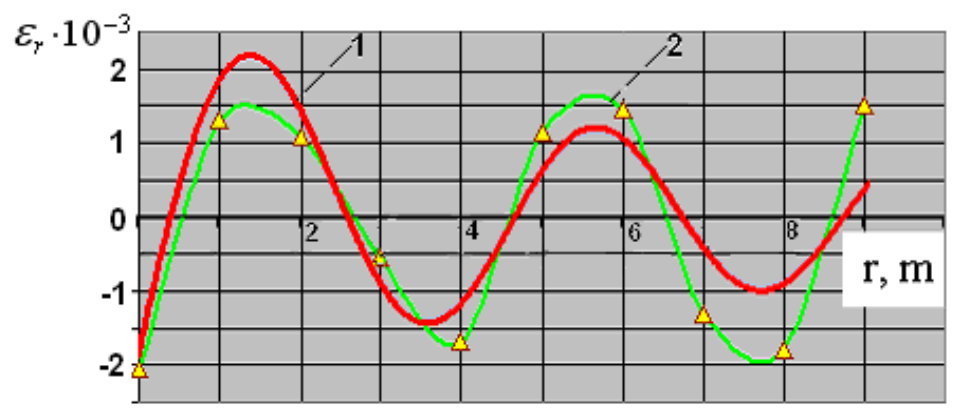

Figure 5 Comparison between theoretical (1) and experimental (2) data of radial deformations

The comparison of the results of the analytical and experimental studies of the weak rock also shows their good convergence (Table 3). The research on zonal failure of the rock mass was carried out for an unlined opening.

Table 3 Comparison of the theoretical and experimental (Neiman et al. 1991) results (unlined opening)

\begin{tabular}{llllll}
\hline \multirow{2}{*}{ Parameter } & \multirow{2}{*}{ Method } & \multicolumn{4}{l}{ Elements of zonal failure structure } \\
& & Zone 1 & Zone 2 & Zone 3 & Zone 4 \\
\hline \multirow{2}{*}{$\begin{array}{l}\text { Location of the furthest zone } \\
\text { boundary, } r / r_{0}\end{array}$} & Experiment & 1.03 & 2.23 & 3.4 & 4.45 \\
\cline { 2 - 6 } & Theory & 1.28 & 2.17 & 3.09 & 3.97 \\
\cline { 2 - 6 } & Deviation (\%) & 24.3 & -2.7 & -9.1 & -12.6 \\
\multirow{2}{*}{$\begin{array}{l}\text { Relative critical zone } \\
\text { stresses, } \sigma / \sigma_{C}^{\text {res }}\end{array}$} & Experiment & 1.1 & 2.2 & 2.7 & - \\
& Theory & 0.95 & 2.1 & 3.1 & - \\
\hline & Deviation (\%) & -13.9 & -4.5 & 14.8 & - \\
\hline
\end{tabular}

It was determined that the basic factor that influences the parameters of zonal failure structure is the value of stresses that act within the rock mass (opening depth). With the increase of stresses, the number of failure zones increases and their radial length increases until it reaches neighbouring zones. The closer the zone is located to the opening boundary, the faster the process. The boundary of the last zone of failure moves further into the rock mass (Figure 6(a)).

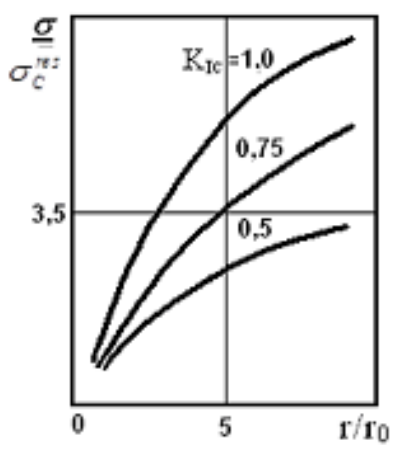

(a)

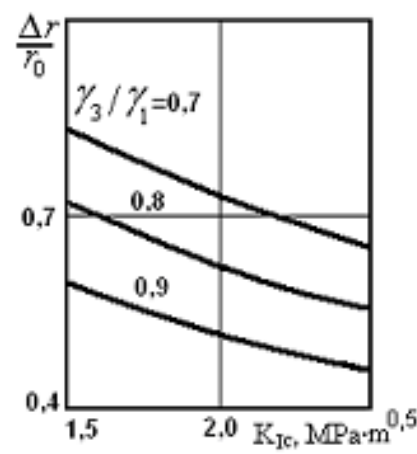

(b)

Figure 6 Dependence of the position of the last failure zone on the relative stresses acting within the rock mass (a) and the radial length of the first zone of failure on the rock fracture toughness (b) 
The parameters of the fracture structure of the rock mass also influence the character of zone failure. The radial length of the zones of failure decreases if the rock fracture toughness rises (Figure $6(b))$. When the rock fracture toughness decreases, zones of failure appear at lower relative stresses and the distance of the last failure zone from the opening boundary increases.

The activity of rock destruction also has a strong influence on the parameters of the zone failure structure. As the fracture faults length increases, the radial length of failure zones increases. This parameter decreases if the dependence $\gamma_{3} / \gamma_{1}$ rises. Regularities determined for weak rock mentioned above are also true for solid rock.

\section{Conclusion}

The research conducted shows that as the depth of the opening rises the zonal character of rock failure becomes more expectable, which should be taken into consideration when designing a lining for such conditions.

A method for determining the mathematical model parameters of zonal failure structure near to deep openings has been developed. A full quality and good quantitative correlation between theoretical forecasting and experimental research has been achieved.

\section{Acknowledgement}

The authors are grateful to Mikhail Guzev for constructive discussions and to Johan Wesseloo and Boris Tarasov for very useful suggestions during the paper preparation. The paper was supported by the grant of Ministry of the Russian Federation Grant No.5.2535.2014K and the grant of RFBR No.26 16-35-00122\16.

\section{References}

Adams, GR \& Jager, AJ 1980, 'Petroscopic observation of rock fracturing ahead of stop faces in deep-level gold mines', Journal of the South African Institute of Mining and Metallurgy, vol. 80, no. 6, pp. 204-209.

Cumming-Potvin, D, Wesseloo, J, Jacobsz, SW \& Kearsley, E 2016, 'Fracture banding in caving mines', Journal of the South African Institute of Mining and Metallurgy, vol. 116, no. 8, pp. 753-761.

Glushihin, FP, Kuznetsov, GN, Shklyarskiy, MF, Pavlov, VN \& Zolotnikov, MS 1991, Modelling in Geomechanics, Nedra Publishing, Moscow, Russia.

Guzev, MA \& Makarov, VV 2007, Deformation and Failure of High Stressed Rocks Around the Openings, Dalnauka Publishing, Vladivostok, Russia.

Guzev, MA \& Paroshin, AA 2000, 'Non-euclid model of zone disintegration of rocks around the underground openings', Journal of Applied Mechanics and Technical Physics, no. 3, pp. 181-195.

Guzev, MA, Makarov, VV \& Ushakov, AA 2005, 'Modeling elastic behavior of compressed rock samples in the pre-failure zone', Journal of Mining Science, Springer, vol. 41, no. 6, pp. 497-509.

Kadich, A \& Edelen, D (eds) 1983, A Gauge Theory of Dislocations and Disclinations, Springer Berlin Heidelberg Publishing, Heidelberg, Germany, pp. 293.

Lisjak, A, Tatone, B, Mahabadi, O \& Grasselli, G 2012, 'Block caving modelling using the Y-Geo hybrid finite-discrete element code', in Proceedings of the 6th International Conference and Exhibition on Mass Mining (MassMin 2012), Canadian Institute of Mining, Metallurgy and Petroleum, 10-14 June 2012, Sudbury.

Makarov, PV 2004, 'About the hierarchical nature of deformation and destruction of firm bodies', Physical Mesomechanics, vol. 7, no. 4, pp. 25-34.

Makarov, VV, Guzev, MA, Odintsev, VN \& Ksendzenko, LS 2016, 'Periodical zonal character of damage near the openings in highly-stressed rock mass conditions', Journal of Rock Mechanics and Geotechnical Engineering, vol. 8, no. 2, pp. $164-169$.

Makarov, VV, Ksendzenko, LS, Opanasyuk, NA \& Golosov, AM 2013, 'Periodic character of failure near openings in high-stress rock mass conditions', in M Kwasniewski and D Lydzba (eds), Proceedings of the 2013 ISRM International Symposium - EUROCK 2013, 21-26 September 2013, Wrocław, pp. 519-523.

Makarov, VV, Ksendzenko, LS, Sapelkina, VM, Opanasyuk, NA, Jashkova, EN \& Voronczova 2007, 'Periodical character of failure near the openings in high depth conditions', The Role of Geomechanics in the Stability of Development of Mining Industry and Civil Engineering: Proceedings of the International Geomechanics Conference, 11-15 June 2007, Nessebar, pp. 107-115.

Metlov, LS, Morozov AF \& Zborshchik MP (2002) Physical Foundations of Mechanism of Zonal Rock Failure in the Vicinity of Mine Working, Journal of Mining Science, vol. 38, iss. 2, pp. 150-155.

Mirenkov, VE 2014, 'Zonal disintegration of rock mass around an underground excavation', Journal of Mining Science, vol. 50, no. 1, pp. 33-37. 
Neiman, LK, Reva, VN, Shmigol, AV \& Kirichenko, VJ 1991, Opening Support on BC "Pavlogradcoal" Mines, CNIEIcoal review Publishing, Moscow, pp. 80.

Odintsev, VN 1996, Rupture Destruction of a Brittle Rocks Mass, IPKON The Russian Academy of Sciences Publishing, Moscow, pp. 166.

Panin, VE 1990, 'The wave nature of plastic deformation of firm bodies', News of High Schools Physics, vol. 33, no. 2, pp. 4-18.

Panin, VE, Grinjaev, JV \& Danilov, VI 1990, Structural Levels of Plastic Deformation and Destruction, Science Publishing, Novosibirsk, Russia, pp. 255.

Qian, QH, Zhou, XP, Yang, HQ, Zhang, YX \& Li, XH 2009, 'Zonal disintegration of surrounding rock mass around the diversion tunnels in Jinping II Hydropower Station, Southwestern China', Theoretical and Applied Fracture Mechanics, vol. 51, no. 2, pp. 129-138.

Reva, VN \& Tropp, EA 1995, 'Elastic-plastic model of zone disintegration of a vicinity of underground excavations', Proceedings of Physicist and the Mechanic of Rocks Failure, VNIMI Publishing, Saint Petersburg, pp. 125-130.

Shemyakin, El, Fisenko, GL, Kurlenja, MV \& Oparin, VN 1986, 'Zone disintegration of rocks around underground excavations, Part 1: The data of natural supervision', Journal of Mining Science, no. 3, pp. 3-15.

Shemyakin, El, Fisenko, GL, Kurlenja, MV \& Oparin, VN 1987, 'Zone disintegration of rocks round underground excavations, Part 3 : Theoretical representations', Journal of Mining Science, no. 1, pp. 3-8.

Xu, NX 2009, 'Identifying rock blocks based on hierarchical rock-mass structure model', Science in China Series D: Earth Sciences, vol. 52, iss. 10, pp. 1612-1623.

\section{Appendix}

Due to the polar symmetry of the task the equilibrium equations are as follows:

$$
\frac{\partial \sigma_{r r}}{\partial r}+\frac{1}{r}\left(\sigma_{r r}-\sigma_{\varphi \varphi}\right)=0, \sigma_{r \varphi}=0, r_{0} \leq \mathrm{r}<\infty,
$$

where:

$$
\begin{aligned}
& \sigma_{r r}=\text { the normal radial stress. } \\
& \sigma_{\varphi \varphi}=\text { the normal tangential stress. } \\
& \sigma_{r \varphi}=\text { the shear stress. }
\end{aligned}
$$

At the opening boundary $\left(r=r_{0}\right)$ and at infinity, the following applied forces exist:

$$
\sigma_{r r}=0 \text { at } r=r_{0} ; \sigma_{r r}, \sigma_{\varphi \varphi} \rightarrow \sigma_{\infty} \text { at } r \rightarrow \infty,
$$

where:

$$
\begin{aligned}
& \sigma_{\infty}=\gamma_{n} \cdot H . \\
& \gamma_{n}=\text { the rock density }\left(\mathrm{N} / \mathrm{m}^{3}\right) . \\
& H=\text { the opening depth }(\mathrm{m}) .
\end{aligned}
$$

The rock mass at a great depth is modelled by the structure, where commonly the conditions of deformation compatibility $\varepsilon_{i j}$ are not met:

$$
R=\frac{\partial^{2} \varepsilon_{11}}{\partial x_{2}^{2}}-2 \frac{\partial^{2} \varepsilon_{12}}{\partial x_{1} \partial x_{2}}+\frac{\partial^{2} \varepsilon_{22}}{\partial x_{1}^{2}} \neq 0
$$

The damage parameter $\boldsymbol{R}$ is expressed by the equation (Guzev \& Paroshin 2000):

$$
\Delta^{2} R-\gamma^{2} R=0
$$

where:

$$
\begin{aligned}
& \Delta=\text { the Laplace operator. } \\
& \gamma \quad=\text { the model parameter. }
\end{aligned}
$$


As the task is plane- and axi-symmetrical, Equation (4) in polar coordinates will become:

$$
\left(\frac{\partial^{2}}{\partial r^{2}}+\frac{1}{r} \frac{\partial}{\partial r}\right)^{2} R=\gamma^{2} R \text {. }
$$

The solution of Equation (5) decreasing at $r \rightarrow \infty$ is:

$$
R(r)=a J_{0}(\sqrt{\gamma} r)+b N_{0}(\sqrt{\gamma} r)+c K_{0}(\sqrt{\gamma} r),
$$

where:

$$
\begin{aligned}
& a=-c \cdot \frac{K_{1}\left(\sqrt{\gamma} \cdot r_{0}\right) \cdot N_{1}\left(\sqrt{\gamma} \cdot r^{*}\right)-K_{1}\left(\sqrt{\gamma} \cdot r^{*}\right) \cdot N_{1}\left(\sqrt{\gamma} \cdot r_{0}\right)}{J_{1}\left(\sqrt{\gamma} \cdot r_{0}\right) \cdot N_{1}\left(\sqrt{\gamma} \cdot r^{*}\right)-J_{1}\left(\sqrt{\gamma} \cdot r^{*}\right) \cdot N_{1}\left(\sqrt{\gamma} \cdot r_{0}\right)}, \\
& b=-c \cdot \frac{J_{1}\left(\sqrt{\gamma} \cdot r_{0}\right) \cdot K_{1}\left(\sqrt{\gamma} \cdot r^{*}\right)-J_{1}\left(\sqrt{\gamma} \cdot r^{*}\right) \cdot K_{1}\left(\sqrt{\gamma} \cdot r_{0}\right)}{J_{1}\left(\sqrt{\gamma} \cdot r_{0}\right) \cdot N_{1}\left(\sqrt{\gamma} \cdot r^{*}\right)-J_{1}\left(\sqrt{\gamma} \cdot r^{*}\right) \cdot N_{1}\left(\sqrt{\gamma} \cdot r_{0}\right)},
\end{aligned}
$$

$r_{0}=$ the radius of an excavation $(\mathrm{m})$.

$r \quad=$ the distance from the center of the excavation to a selected point in a rock mass $(\mathrm{m})$.

$J_{0}, J_{1}, N_{0}, N_{1}, K_{0}, K_{1}=$ the Bessel, Neumann, and MacDonald functions of zero and first orders, respectively.

$\gamma, c=$ parameters of mathematical model for the rock mass surrounding a deep-seated excavation are found experimentally, using samples of rocks. 